\title{
Les archives d'Aimé Césaire sur le site André Breton
}

\section{Kora Véron}

\section{OpenEdition}

\section{Journals}

Édition électronique

URL : https://journals.openedition.org/coma/855

DOI : 10.4000/coma.855

ISSN : 2275-1742

\section{Éditeur}

Institut des textes \& manuscrits modernes (ITEM)

\section{Référence électronique}

Kora Véron, «Les archives d'Aimé Césaire sur le site André Breton », Continents manuscrits [En ligne], 8 | 2017, mis en ligne le 15 mars 2017, consulté le 13 janvier 2023. URL : http:// journals.openedition.org/coma/855

Ce document a été généré automatiquement le 13 janvier 2023.

\section{(c) $(1) \&$}

Creative Commons - Attribution - Pas d'Utilisation Commerciale - Pas de Modification 4.0 International - CC BY-NC-ND 4.0

https://creativecommons.org/licenses/by-nc-nd/4.0/ 


\title{
Les archives d'Aimé Césaire sur le site André Breton
}

\author{
Kora Véron
}

\section{Genèse d'un site}

1 La responsable du Site André Breton ${ }^{1}$, Constance Krebs, a raconté l'élaboration de cette base de données évolutive ${ }^{2}$ - sous forme de wiki -, à partir de la collection rassemblée par Breton tout au long de sa vie, dans son atelier du 42, rue Fontaine. Des informations complémentaires figurent sur le site lui-même ${ }^{3}$.

2 Après la mort d'Elisa, dernière épouse de Breton, le 5 avril 2000, la fille de Jacqueline et d'André Breton, Aube Breton-Elléouët, se décide à disperser la collection de son père. Des enchères sont organisées à Drouot par les commissaires-priseurs, Laurence Calmels et Cyrille Cohen. L'État a préempté 335 des 4100 lots. Seize d'entre eux seront finalement offerts par Aube et sa fille, Oona. Le « Mur » d'André Breton a été acquis par le musée national d'Art moderne, en dation, comme paiement des droits de succession ${ }^{4}$. Le site de l'Hôtel Drouot indique ${ }^{5}$ :

La dispersion fleuve menée par la maison de vente Calmels-Cohen s'est terminée le 17 avril avec un produit vendu qui dépasse les 46 millions d'euros, soit $50 \%$ de plus que l'estimation attendue. [...]

Estimée à 30 millions $€$, cette vente historique a atteint des sommets spectaculaires et restera dans la mémoire de tous les amateurs et collectionneurs comme l'événement majeur de ces vingt dernières années sur la place de Paris.

À l'issue des 21 vacations, quelques chiffres illustrent ce succès :

50000 personnes ont visité les 7 salles d'exposition de l'Hôtel Drouot Un catalogue en 8 volumes pesant $10 \mathrm{~kg}$ et un DVD Rom incluant 30000 images scannées, destiné à devenir une base érudite de référence sur le surréalisme, 52 heures de ventes pour disperser les 4100 lots, 2 doubles salles ouvertes aux 2000 enchérisseurs, 30 lignes de téléphone relayaient les enchérisseurs étrangers, 335 préemptions de l'État pour un montant total de 11,8 $\mathrm{M} €$ hors frais et pour plus de 33 institutions françaises, 36 enchères de plus de $150000 €$ dont 7 supérieures à $1 \mathrm{M}$ d'euros. [...]

La vente des seuls manuscrits a rapporté $3646856 €$ (3 $082250 €$ hors frais). 
4 Le volumineux catalogue élaboré pour cette vente, ainsi que les images numérisées à l'initiative de Laurence Calmels ont commencé à alimenter le site intitulé Atelier André Breton, devenu par la suite Site André Breton. Il s'agit d'un work in progress, puisque les images disponibles sont introduites progressivement, et que des précisions sont apportées par de nouveaux contributeurs.

\section{Problèmes de fonds et de forme}

5 Constitué essentiellement pendant la Seconde Guerre mondiale, le fonds Césaire est lacunaire : certaines lettres et certains poèmes ne sont pas parvenus à destination, ou ont été égarés au cours des déménagements des deux interlocuteurs.

6 De plus, une grande partie de la correspondance a été confiée à la Bibliothèque littéraire Jacques Doucet, conformément à la volonté d'André Breton. Les liens entre Breton et Doucet sont bien connus et l'on sait, par exemple, que le couturier avait demandé à Breton et à Aragon, au début de 1922, de classer et compléter sa bibliothèque ${ }^{6}$.

7 Ainsi, plusieurs manuscrits, séparés des lettres qui les accompagnaient, sont difficiles à dater. D'autres, restés mêlés à la correspondance, sont absents du site et doivent être consultés dans le fonds Breton de la Bibliothèque Doucet. On peut espérer qu'un jour les documents seront réunis électroniquement.

8 D'autre part, certaines notices du catalogue de la vente d'avril 2003, reprises sur les deux versions du site, ont été rédigées à la hâte. Celles concernant Césaire comportaient des erreurs relatives aux dates et aux contextes éditoriauxincohérents - attribués à certaines pièces. J'ai proposé quelques corrections à Constance Krebs, qui a accepté de les prendre en compte en juin 2016.

9 Enfin, si le système de visualisation adopté convient aux photographies des œuvres d'art collectionnées par Breton, il se révèle parfois frustrant pour la consultation des manuscrits ou de certains documents dactylographiés, présentés en outre dans un ordre imposé, peu compréhensible (il aurait été pourtant utile de pouvoir sélectionner les documents par ordre chronologique). D'autant que les «vignettes» qui les annoncent sont trop petites pour permettre de distinguer leur contenu, comme l'indique Corinne Welger-Barboza, dans son article: «Le site André Breton - Un monument-document $»^{7}$, publié dans un carnet d'Hypothèses intitulé "L'observatoire critique ».

10 Frustrante aussi l'impossibilité de télécharger les documents. Pour les transcrire et les étudier confortablement, il faut donc passer par une capture d'écran et quelques bricolages qui compliquent le processus.

11 En dépit de ces quelques problèmes, le Site André Breton est extrêmement précieux. On y trouve tout d'abord une grande partie des manuscrits ou des tapuscrits correspondant aux textes qui seront repris dans Les Armes miraculeuses, publié chez Gallimard en avril 1946 : transgénérique, ce recueil est composé de vingt-six poèmes; d'une tragédie, Et les chiens se taisaient ; et d'une " Postface » en prose, intitulée « Mythe ».

12 Les archives disponibles mettent en évidence la genèse tourmentée de ce recueil et l'art poétique combinatoire de Césaire. 


\section{Un art combinatoire} Guerre mondiale. À la fin du mois d'août 1939, juste avant le début des hostilités, le jeune Césaire - il a 26 ans - rentre à la Martinique, avec sa femme, Suzanne, enceinte de leur deuxième enfant, et leur premier fils. Aimé Césaire a passé huit années à Paris : il a intégré l'École de la rue d'Ulm, écrit dans L'Étudiant martiniquais, qu'il a transformé en L'Étudiant noir, et publié une première version de Cahier d'un retour au pays natal dans la revue Volontés.

L'œuvre poétique de Césaire évoque une menace obsessionnelle de coupure, de dislocation, de lacération, de dispersion (Soleil Cou coupé, Corps perdu).

L'écriture, qui rassemble membres et mots, est en rapport avec ce que le poète appelle son «moi profond» :

Je ne cesse d'écrire des poèmes, sans toujours les publier. La poésie est ma raison d'être, mon exutoire, ma bouée de sauvetage. C'est par la poésie que s'exprime mon moi profond, que s'affirme mon être [...] Pour le reste, je me prête à la gesticulation sociale $[. . .]^{8}$.

Le mot est démiurge, c'est lui qui organise le chaos, c'est avec les mots que nous passons de l'existence toute simple à l'être'.

Césaire combine donc les mots pour donner forme et consistance à un être individuel et collectif chaotique, car, pour Césaire, «je » n'est pas « un autre », mais tout un peuple, plus un volcan.

Comment Césaire assemble-t-il ? Il l'explique lui-même :

Tout ce que je peux dire... je n'écris jamais... ça m'est très difficile d'écrire les choses d'un seul tenant - ça m'est très difficile. Je peux encore composer après coup, mais toujours, chez moi, c'est une idée qui vient, c'est un mot que je fixe... vous comprenez, qui indique une tonalité, et ça peut me venir n'importe où, dans le métro. Sur un petit ticket de métro, je peux écrire un mot, puis après je peux l'oublier, vous comprenez. C'est toujours comme ça que j'ai fait, et alors après, bien sûr, c'est noté, c'est fixé, et j'en fais un petit peu un montage. Après. C'est comme ça que ça se présente chez moi. D'abord un sentiment intense, premier, que je fixe le plus rapidement possible, le plus immédiatement possible ${ }^{10} \ldots$

Son écriture procède par amplification, à partir d'un mot qu'il «fixe ", et "après ", seulement après, il élabore " un petit peu un montage ", c'est-à-dire une combinaison hétéroclite de "fragments", toujours susceptible d'être recombinée, contrairement aux collages dadaïstes ou surréalistes qui, eux, restent stables. Pour résumer, disons que Césaire, conformément aux injonctions de son moi profond, a fait du "copié-collé " toute sa vie de poète, sans même se douter qu'un tel procédé informatique avait été inventé, car il utilisait des ciseaux et de la colle.

\section{Rencontre en eaux troubles}

Cette tendance générale est accentuée pendant la période correspondant à la Seconde La «drôle de guerre " est déclarée en septembre 1939. Elle perdurera aux Antilles françaises, comme variante tropicalisée et édulcorée d'un régime de collaboration, sous étroit contrôle des États-Unis ${ }^{11}$. Ce n'est pas drôle, mais ce n'est pas vraiment la guerre. La Martinique, bien loin des conflits armés, rejoindra la France libre en juillet 1943. En 
attendant, l'amiral Georges Robert commande les forces navales de l'Atlantique Ouest depuis la Martinique. Il y promeut Vichy, notamment en révoquant les pouvoirs locaux après l'armistice de 1940, ou en organisant des manifestations sportives, culturelles et religieuses à la gloire du régime de Pétain, tout en se montrant soucieux d'assurer aux territoires qu'il contrôle une certaine neutralité qui garantisse l'intégrité de la flotte de guerre et celle du stock de 286 tonnes d'or de la Banque de France évacué à la Martinique.

21 Après son retour à Fort-de-France, Césaire avait fait paraître un premier hommage à Charles Péguy, dans L'Action socialiste (octobre 1939) ${ }^{12}$. Puis rien d'autre pendant un an et demi. On comprend que l'heure était grave et qu'il lui fallait du temps pour rebondir. En avril 1941, il lance Tropiques, avec sa femme, Suzanne, son beau-frère, Aristide Maugée, quelques collègues du Lycée Schœlcher, dont le professeur de philosophie, René Ménil. Destinée à combattre un « vide culturel »" la revue, modestement diffusée, constitue aussi pour Césaire un laboratoire qui lui permet d'expérimenter ses combinaisons poétiques.

Or, peu après la sortie du premier numéro de Tropiques, le 24 avril 1941, le Capitaine Paul Lemerle arrive à Fort-de-France, en provenance de Marseille, après un mois d'une traversée éprouvante. Les réfugiés qui débarquent à la Martinique pour quelques semaines trouveront ensuite refuge aux États-Unis, au Canada, au Mexique, à Cuba...

À son bord, une foule d'anonymes et quelques personnalités, dont André Breton, accompagné de sa femme, Jacqueline, et d'Aube, sa fille; Wifredo Lam et sa femme Helena ; Claude Lévi-Strauss ; Victor Serge et son fils Vlady ; Anna Seghers et sa famille. La famille Masson arrive une semaine plus tard à la Martinique à bord d'un autre bateau.

Les circonstances de cette avenue of escape sont bien documentées, je n'y reviens pas. Je ne reprends pas davantage ici les circonstances de la rencontre entre Césaire et Breton à Fort-de-France, mais pour une raison inverse : il faudrait en étudier attentivement les circonstances, au-delà de ce que Breton a pu en écrire dans Martinique charmeuse de serpents ${ }^{14}$. Ce qui est sûr, c'est que les deux hommes se fascinent réciproquement. Le séjour sur une île "primitive " et magique, en compagnie d'un "cannibale $»^{15}$ assez normalien pour soutenir une conversation poétique aura été un réconfort et une puissante source d'inspiration pour Breton. Et le jeune Césaire, à la recherche d'un nouvel élan, va bénéficier du rayonnement surréaliste de son nouvel ami.

Arrivé à New York fin mai 1941, Breton désire insuffler une nouvelle vie à son mouvement. Une nouvelle recrue et de nouveaux mythes s'imposent dans l'adversité de l'exil. Il engage alors une correspondance avec Césaire et lui propose généreusement d'éditer ses poèmes à New York, à la première occasion, comme l'indique une lettre de Suzanne Césaire adressée à André Breton, datée du 21 octobre 1941: "Vous devinez sans peine l'émotion d'Aimé à l'idée d'être publié par vous. Il s'en remet entièrement à vous pour tout ce qui concerne la publication. Il joint à cette lettre des poèmes inédits. » 


\section{Avant-textes de Les Armes miraculeuses}

\section{Cinq manuscrits ${ }^{16}, 10$ janvier 1943}

27 Les premiers poèmes sont envoyés le 10 janvier 1943, avec une lettre adressée à André Breton, dans laquelle Aimé Césaire écrit: "Je vous envoie 5 poèmes dont vous disposerez à votre guise ".

"Annonciation » (dédié à André Breton), "Tam-tam I» (dédié à Benjamin Péret) et «Tam-tam II » (dédié à Wifredo [Lam]) seront publiés dans le numéro de $V V V, \mathrm{n}^{\circ}$ 2-3, de mars 1943. "Légende » et "Tendresse » seront intégrés au poème "Les pur-sang ", dans l'édition du recueil Les Armes miraculeuses, publiée en avril 1946.

\section{Colombes et menfenil17}

Ce dossier est complexe, et la date erronée en provenance du catalogue de la vente Breton (1945) a achevé d'embrouiller quelques chercheurs. Seul Alex Gil la contestait avec moi $^{18}$.

Le 3 août 1943, Aimé Césaire avait écrit à André Breton : «Cher ami, merci, merci pour tout : pour votre amitié, votre affection, votre sollicitude; merci pour l'offre que vous me faites d'éditer mes poèmes - je tâcherai de les réunir au plus vite.» Le 22 septembre 1943, Césaire envoie un " poème " : très certainement la version de "Les pur-sang " qui sera publié dans Hémisphères $\mathrm{n}^{\circ} 2-3$, en novembre; et de la deuxième version du poème "Simouns "; promettant à Breton le "drame nègre " qu'il vient de terminer. Le 16 novembre 1943, il confirme à Breton l'envoi de son poème "Batouque ", et ajoute: "Je vous enverrai bientôt par paquet-poste les manuscrits d'un recueil possible de poèmes ainsi qu'un drame : Et les chiens se taisaient. »

31 Je pense que ce "recueil» n'a pas été envoyé par la poste. J'avais montré précédemment qu'une nouvelle version de Cahier d'un retour au pays natal ${ }^{19}$, et la première version de Et les chiens se taisaient ${ }^{20}$ avaient été confiées à Henri Seyrig. Nommé conseiller culturel de la France libre à New York par le général de Gaulle en 1942, Seyrig mène des missions à travers les Amériques et la Caraïbe. De novembre 1942 à mai 1943, il a parcouru l'Amérique latine. Son séjour aux Antilles françaises s'est déroulé du 10 octobre au 17 novembre $1943^{21}$. Seyrig quitte donc la Martinique juste au moment où Césaire est prêt à envoyer son " paquet-poste ", et il ne sera plus question de cet envoi par la suite dans la correspondance entre Césaire et Breton : l'affaire est réglée. La concordance des dates et l'analyse des lettres de Césaire permettent d'affirmer que Seyrig a aussi transporté avec lui Colombes et menfenil, ainsi que Le Grand Midi, alias Tombeau du Soleil.

32 Cela signifie qu'en novembre 1943, Césaire considère comme achevés : sa nouvelle version de Cahier d'un retour au pays natal destinée à une édition bilingue américaine (chez Hémisphères); un "drame nègre» dont le héros se nomme Toussaint Louverture; et son premier recueil de poèmes. Or, rien ne se déroulera comme prévu. Ces trois œuvres seront recombinées pendant les années suivantes, le drame et le recueil étant intégrés à Les Armes miraculeuses, tandis qu'une nouvelle version du Cahier paraîtra en mars 1947, chez Bordas, quelques semaines après l'édition new-yorkaise de Brentano's, en janvier. 
2. « N'ayez point pitié de moi ».

3. «Survie».

4. « Poème pour l'aube ».

5. « Poème » (barré).

6. «Tam-tam de nuit ».

7. « Tam-Tam I ( ( voir VVV n 2 »).

8. « Tam-Tam II ( (« voir VVV n 2 »).

9. «Annonciation » (« voir VVV n 2 »).

10. «Femme d'eau ».

11. «Simouns" («texte déjà communiqué »).

12. «Batouque».

$1 /$ de poèmes parus préalablement dans :

- Tropiques $\mathrm{n}^{\circ}$ 3, en octobre $1941:$ Au delà (p. 2), « N'ayez point pitié de moi » (p.3), Survie (p.4).

- Tropiques $\mathrm{n}^{\circ} 4$, en janvier 1942 : Poème pour l'aube (p.5)

- Tropiques n 6-7, en février 1943 : «Tam-tam de nuit » (p. 7), « Femme d'eau » (p. 9)

$2 /$ de références à des textes parus dans le $V V V n^{\circ} 2-3$, de mars 1943, et déjà envoyés à André Breton dans Cinq manuscrits: "Tam-tam I», «Tam-tam II» (p. 8), «Annonciation », (p. 8).

$3 /$ de poèmes inédits

- un « poème » (p. 6) qui deviendra « Colombes bruissement du sang... » dans Leitmotiv (Santiago du Chili), $n^{\circ}$ 2-3, décembre 1943-janvier 1944, puis « Soleil serpent » à partir de sa publication dans Hémisphères $\mathrm{n}^{\circ} 4$.

- une référence à "Simouns » (p. 10), qui prendra le titre de "Les Oubliettes de la mer et du déluge » dans la revue Fontaine, en mars 1946.

- «Batouque » (11), dont une version sera publiée dans VVV, nº 4, en février 1944.

Sept poèmes («Au delà »; "N'ayez point pitié de moi »; "Survie »; "Poème pour l'aube »; «Soleil serpent »; «Tam-tam de nuit »; «Femme d'eau »), regroupés sous le titre Colombes et menfenil, paraîtront dans le ${ }^{\circ} 4$ d'Hémisphères, pendant l'été 1944. 

nouveau fâché avec Breton, après l'échec de l'édition de Cahier d'un retour au pays natal aux éditions Hémisphères. Le projet était bien avancé, mais Goll ne disposait plus de papier pour imprimer de nouveaux ouvrages ${ }^{23}$. La querelle entre Goll et Breton, provisoirement apaisée pendant quelques mois lors de leur exil new-yorkais, datait cependant de 1923-1924, moment où les deux hommes s'étaient violemment affrontés autour du legs surréaliste d'Apollinaire. Breton avait gagné la bataille en publiant son premier « Manifeste $»^{24}$.

Césaire ne semble pas avoir été informé des accords éditoriaux, puis des dissensions entre Breton et Goll, puisqu'il écrit à Breton, le 2 avril 1945 : «À propos, je n'ai aucune espèce de relation avec Goll. J'ignore ce monsieur. J'ignorais même qu'il détînt mes poèmes, et mon drame. » Goll veillera toutefois à ne pas reprendre dans Hémisphères les poèmes édités par Breton dans VVV (« Tam-tam I », « Tam-tam II », « Annonciation » et «Batouque »), et ne dispose pas de «Simoun » (texte déjà communiqué).

\section{Tombeau du soleil}

On trouve dans Tombeau du soleil douze « fragments ».

1. «Les pur-sang », première partie de «Fragments d'un poèmes » de Tropiques $\mathrm{n}^{\circ} 1$ (avril 1941).

2. «Investiture».

3. Un texte sans titre commençant par « Parce que les jardins timbrés ».

4. «Parce que mon beau pays » (version augmentée d'un extrait de «Fragments d'un poème Le Grand Midi » de Tropiques $n^{\circ} 2$, en juillet 1941).

5. Un texte sans titre commençant par « ô retour, ô paupières ".

6. Un texte sans titre commençant par « Mais qui m'a mené ici » qui correspond à une partie de « Histoire de vivre », publié dans Tropiques $\mathrm{n}^{\circ} 4$ (janvier 1942).

7. Un texte sans titre commençant par "ô Chimborazo violent ", qui correspond à « Tendresse » dans Cinq manuscrits.

8. Un texte sans titre commençant par: "Et les Et les collines soulevèrent », qui correspond à une partie de « Histoire de vivre ", publié dans Tropiques $\mathrm{n}^{\circ} 4$.

9. «Calcination » montage du début de «Fragments d'un poème Le Grand Midi » de Tropiques $\mathrm{n}^{\circ} 2$, et de « Fragments d'un poème » de Tropiques $\mathrm{n}^{\circ} 1$.

10. «Miroir fertile » suite de « Fragments d'un poème » de Tropiques $\mathrm{n}^{\circ} 1$.

11. «Le Grand Midi » version modifiée de «Fragments d'un poème, Le Grand Midi (Fin) ", publié dans Tropiques $n^{\circ} 2$.

12. «Conquête de l'aube» renvoi à $V V V \mathrm{n}^{\circ} 1$, de juin 1942 (alors accompagné de l'indication : « Extraits inédits du Grand Midi »), avec quelques corrections.

La maquette a été manifestement élaborée rapidement, sur des feuilles hétéroclites recyclées. Elle est constituée d'extraits des numéros 1,2 et 4 de Tropiques, déchirés, collés, et corrigés manuellement $(1,10,11)$; de manuscrits intercalés (2 à 9$)$; et d'un renvoi à $V V V n^{\circ} 1$ (12). Les titres sont ajoutés à la main, sans que l'on puisse toujours savoir de quelle main ; et la numérotation des poèmes est instable.

Contrairement à Colombes et menfenil, Césaire désire donner une unité à ces fragments qui ne devaient former qu'un seul poème : Tombeau du soleil, alias Le Grand Midi. Sans y parvenir. Tombeau $d u$ soleil ne verra pas le jour. Certains fragments resteront inédits, 
tandis que les autres s'intégreront aux poèmes du recueil Les Armes miraculeuses ou de Corps perdu ${ }^{26}$.

\section{IV « La forêt vierge » et « Les armes miraculeuses » ${ }^{27}$}

Comme je l'indique sur le Site André Breton, il n'est pas possible de dater précisément les manuscrits de "La forêt vierge ", poème au destin tumultueux, qui sera recombiné et divisé plusieurs fois selon les éditions, avec la suppression de nombreux passages.

Le deuxième manuscrit, "poème", prendra le titre de "Les armes miraculeuses", quand il sera publié dans le recueil Les Armes miraculeuses.

Dans les documents conservés par la Bibliothèque Doucet, on trouve notamment un "Poème » qui commence par "Ma grande statue blessée ", envoyé avec une lettre datée du 2 avril 1945, et qui sera intitulé «L'irrémédiable ».

Enfin, certains manuscrits confiés à Ivan Goll n'ont pas été restitués à André Breton. Ils sont conservés à bibliothèque municipale de Saint-Dié des Vosges. Il s'agit d'une première version de la pièce de théâtre Et les chiens se taisaient, ainsi que d'un tapuscrit comprenant quelques poèmes de Tombeau du soleil ${ }^{28}$.

Le tableau de correspondances entre les manuscrits-tapuscrits, les poèmes publiés en revue, et les volumes serait trop long à commenter ici, mais on constate facilement que le recueil Les Armes miraculeuses est majoritairement composé de textes recombinés à partir de ceux ayant été communiqués à André Breton, à l'exception de :

. «Avis de tir»

. «Le cristal automatique»

. «Visitation»

. «Perdition»

. «Postface : Mythe»

et parmi eux, seuls :

. «Le cristal automatique»

. «Visitation »

. «Postface : Mythe»

sont vraiment nouveaux, les autres ayant été publiés dans Tropiques.

En revanche, certains textes figurant dans le dossier Breton (site et autres archives) n'ont pas été publiés préalablement au recueil et peuvent sembler faussement nouveaux :

. «Phrase»

. «Investiture»

. « La forêt vierge »

\section{Archives croisées}

Le Site André Breton ne se limite pas aux avant-textes de Les Armes miraculeuses. On y trouve également de nombreuses archives éclairant les liens entre Breton et Césaire. Je ne prends que trois exemples. 


\section{1-Deuxième passage d'André Breton à la Martinique, $1946^{29}$} alors qu'il accorde un long entretien au poète et critique d'art Nicolas Calas, pour la revue View ${ }^{36}$, en 1941. Calas lui demande s'il a déjà rêvé d'Hitler. Breton répond que non, mais ajoute que lors de son escale en République dominicaine, après son premier séjour martiniquais, il a rêvé qu'il était Zapata, préparant son armée à recevoir Toussaint Louverture afin de lui rendre, le lendemain, les honneurs qui lui revenaient :

In an ambitious way, not usual with me, I dreamt while in the throes of a great exaltation at Ciudad Truillo [lors de son escale en République dominicaine] that I was Zapata, making ready with my army to receive Toussaint Louverture the following day and to render him the honor to which he was entitled. (p. 1) Césaire avait donc certainement parlé à Breton de la première version de sa pièce Et les 
chiens se taisaient, dont Toussaint Louverture était le héros tragique, lors de leurs conversations d'avril et de mai 1941, à la Martinique. L'ombre de Desnos ne s'est-elle pas glissée également dans ce rêve?

\section{NOTES}

1. http://www.andrebreton.fr/

2. Constance Krebs: https://amontour.wordpress.com/2009/11/05/site-breton-de-ledition-enligne/

3. http://www.andrebreton.fr/card/offrir_le_surrealisme_au_monde

4. Le Nouvel Observateur, 1-7 mai 2003.

5. http://www.drouot.com/static/drouot_resultat_detail.html?idActu=7314

6. http://www.andrebreton.fr/work/56600100281870

7. https://observatoire-critique.hypotheses.org/1252\#footnote_9_1252

8. « Aimé Césaire, nègre rebelle » [entretien avec Philippe Decraene], Le Monde, 6 décembre 1981 : Kora Véron, Thomas Hale, Les Écrits d'Aimé Césaire : biobibliographie commentée, 1913-2008, Paris, Honoré Champion, (Poétiques et esthétiques, $\mathrm{Xx}^{\mathrm{e}}-\mathrm{XxI}^{\mathrm{e}}$ siècle), 2 volumes, mai 2013, p. 552. Cet ouvrage constitue une nouvelle version - corrigée et augmentée - de la version de Thomas Hale, parue en 1978.

9. «Aimé Césaire : Où que j'aille je reste un nègre déraciné des Antilles » [entretien avec Pierre Boncenne], Lire, $\mathrm{n}^{\circ}$ 87, novembre 1982 : Les Écrits, p. 566.

10. «Entretien avec Aimé Césaire, Fort-de-France, le 14 février 1973 » [Michel Benamou], Cahiers césairiens, The Pennsylvania State University, $\mathrm{n}^{\circ} 1$, printemps 1974, p. 4-8: Les Écrits, p. 455. https://collection1.libraries.psu.edu/cdm/compoundobject/collection/ces/id/56

11. Voir notamment : Léo Élisabeth, «Vichy aux Antilles et en Guyane : 1940-1943 », Outre-mers, tome XCI, $\mathrm{n}^{\circ} 342-343,1^{\mathrm{er}}$ semestre 2004, p. 145-174.

12. Les Écrits, p. 35.

13. Entretien avec Jacqueline Leiner qui introduit la réédition de Tropiques en 1978, aux éditions Jean-Michel Place.

14. André Breton et André Masson, aux Éditions du Sagittaire en 1948 ; réédition chez JeanJacques Pauvert en 1972.

15. «Parce que nous vous haïssons, vous et votre raison, nous nous réclamons de la démence précoce, de la folie flambante, du cannibalisme tenace. ", «En guise de manifeste littéraire » [dédié à André Breton], Tropiques, nº 5, avril 1942, p. 7-12.

16. http://www.andrebreton.fr/fr/item/?GCOI=56600100218250

17. http://www.andrebreton.fr/fr/item/?GCOI=56600100037610

18. Alex Gil, «Focus génétique sur Les Armes miraculeuses d'Aimé Césaire» : https:// coma.revues.org/254

19. Kora Véron, "Césaire at the Crossroads in Haiti : Correspondance with Henri Seyrig ", Comparative Literature Studies, volume 50, $\mathrm{n}^{\circ} 3$, p. 430-444. Pennsylvania State University Press, août 2013, p. 430-444.

20. Kora Véron, «Configurations d'Aimé Césaire : pour une biotique connectée ». Conférence du 25 novembre 2016, dans le cadre du séminaire de l'équipe «manuscrit francophone » de l'Item, École normale supérieure de la rue d'Ulm. 
21. Journal d'Henri Seyrig, archives privées.

22. "Allocution de M. Césaire, maire de Fort-de-France ", Journal officiel de la Martinique, ${ }^{\circ}{ }^{\circ} 26$, 21 juin 1945, p. 353-354 : Les Écrits, p. 78-79.

23. Le 2 mai 1944, Goll écrit à Breton : «J'ai toujours eu l'intention de publier aux « Éditions Hémisphères ", une édition bilingue du "Cahier d'un Retour au Pays Natal" et je comprends votre méfiance concernant ma nouvelle attitude. J'ai l'air d'un usurpateur, c'est vrai, hélas ce que vous ne savez pas c'est que depuis quelques semaines, au courant desquelles il ne m'a pas été donné de vous voir le W P B m'a refusé l'allocation du papier nécessaire pour ce livre et quelques autres. » http://gollyvanetclaire.canalblog.com/archives/2008/12/05/11640923.html.

Le War Production Board est une agence du gouvernement américain chargée de superviser la production industrielle pendant la Seconde guerre. Elle établit des priorités dans la distribution d'essence, de métaux, de papier, etc.

24. Albert Ronsin, "Yvan Goll et André Breton. La querelle littéraire à propos de Surréalisme », Europe, $n^{\circ} 899$, mars 2004, p. 191-209. Voir aussi : Stephen Steele, Nouveaux regards sur Ivan Goll en exil avec un choix de ses lettres des Amériques. Tübingen : Narr, 2010.

25. http://www.andrebreton.fr/fr/item/?GCOI=56600100522190

26. Paris : Éditions Fragrance, 3 juin 1950, avec trente-deux gravures de Pablo Picasso.

27. http://www.andrebreton.fr/fr/item/?GCOI=56600100902670

28. Alex Gil, Migrant Textuality : On the fields of Aimé Césaire's Et les chiens se taisaient, 2012 ; et Kora Véron, conférence du 25 novembre 2016.

29.

http://www.andrebreton.fr/work/56600100746270?

back_rql=Any\%20X\%20ORDERBY\%20FTIRANK\%28X\%29\%20DESC\%20WHERE\%20X\%20has_text\%20\%22martinique\%22\&bac

30.

http://gallica.bnf.fr/ark:/12148/bpt6k326310/

f6.image.r=l\%C3\%A9gitime\%20defense.langFR//

31.

http://www.andrebreton.fr/work/56600100627580?

back_rql=Any\%20X\%200RDERBY\%20FTIRANK\%28X\%29\%20DESC\%20WHERE\%20X\%20has_text\%20\%22martinique\%22\&back_url=http\%3

32. http://www.andrebreton.fr/work/56600100650201

33. Catalogue présenté par André Breton et Marcel Duchamp. Maeght éditions (Pierre à feu), 1947, p. 76-77 : http://www.andrebreton.fr/series/81

34. K éditeur (Le Quadrangle), 23 avril 1948, p. 79-81.

35. http://www.andrebreton.fr/work/56600100167210

36. Déclinée en Through the Eyes of Poets. Le journal avait été fondé en septembre 1940 par Charles Henri Ford. L'entretien, qui constitue la première intervention de Breton dans la presse américaine, sera publié dans le numéro 7-8 en octobre-novembre 1941. Breton a repris ensuite le texte en indiquant qu'il s'agit d'un entretien avec Charles-Henri Ford, et en supprimant certains passages, dont celui-ci Il est ajouté en note dans les Euvres complètes (Gallimard «Bibliothèque de la Pléiade », tome III, 1999, p. 1316), mais l'identité de l'interlocuteur n'est pas rectifiée.

\section{RÉSUMÉS}

Le Site André Breton offre aux chercheurs des ressources exceptionnelles sur la constellation surréaliste : par leur nombre, leur variété, leur intérêt. Les documents concernant Aimé Césaire, 
relatifs à une période allant surtout de 1942 à 1948, sont d'autant plus précieux que le poète martiniquais n'a conservé lui-même que très peu d'archives. Ils révèlent l'art poétique combinatoire de Césaire, à travers la genèse tourmentée du recueil Les Armes miraculeuses, et permettent d'apprécier le lien entre le jeune poète et son aîné, en exil dans le Nouveau Monde.

INDEX

Mots-clés : Césaire Aimé, site André Breton, Ivan Goll, Henri Seyrig, Cahier d'un retour au pays natal, Et les chiens se taisaient, Les Armes miraculeuses

\section{AUTEUR}

\section{KORA VÉRON}

Chercheuse associée, équipe « Manuscrits francophones », ITEM (UMR 8132, CNRS-ENS) 